\title{
Systemic vaccination induces clonally diverse SIV-specific CD8 + T-cell populations in systemic and mucosal compartments
}

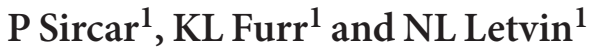

An HIV-1 vaccine must elicit a clonally diverse virus-specific CD8 + T-cell response to contain mutant virus forms, and these responses must be present in mucosal tissues, which are the site of early HIV-1 replication. We show that systemic delivery of prototype vaccine vectors in rhesus monkeys induced SIV (simian immunodeficiency virus)-specific CD8+ T-cell responses in systemic and mucosal compartments with comparable clonal compositions. Although clonal sharing was maintained between the peripheral blood and lungs, the clonal constituents of the vaccine-induced CD8 + T-cell populations in the gastrointestinal mucosal tissues evolved away from the peripheral blood population. A phenotypic characterization indicated that the divergence was a consequence of differential trafficking and retention of the vaccineinduced cells in mucosal compartments. These findings highlight the circulation of vaccine-induced CD8 + T-cell populations between systemic and mucosal compartments and the importance of the expression of specific homing molecules for localization in mucosal tissues.

\section{INTRODUCTION}

Mucosal tissues have a central role in early HIV-1/SIV (simian immunodeficiency virus) pathogenesis. Virus transmission usually occurs across genital mucosal surfaces, and the intestinal mucosa is the site of early viral replication and dramatic CD $4+$ CCR5 + T-cell depletion. Therefore, it is likely that successful vaccination strategies will need to induce effective mucosal immunity. ${ }^{1-4}$ However, there are divergent reports on whether a systemic or mucosal route of vaccine delivery for HIV-1/SIV is optimal for generating mucosal responses. ${ }^{2,5,6}$

Although virus-specific CD8 + T cells have a critical role in the immune defense against HIV-1/SIV replication, a number of critical challenges remain in inducing immune responses that can control these rapidly mutating viruses. ${ }^{7,8}$ Clonal diversity will be an important determinant of CD8 + T-cell-mediated immune protection because T-cell antigen receptor (TCR) diversity in an epitope-specific cellular immune response should facilitate the recognition of emerging viral variants during infection and increase the presence of high-avidity CD $8+$ T cells. ${ }^{9-12}$ It is unclear whether the CD8 + T-cell TCR repertoire can be stimulated by different vaccine regimens to generate this clonal diversity.
We have previously demonstrated that the clonal repertoire of an immunodominant epitope-specific CD8 + T-cell response is shared in mucosal tissues and peripheral blood of chronically SIV-infected rhesus monkeys. ${ }^{13}$ In the present study, we evaluated the clonal repertoire of epitope-specific CD8 + T-cell responses generated by various prime-boost vaccination regimens. Furthermore, we wanted to determine whether systemic prime-boost immunization with recombinant vectors can generate clonally diverse, long-term responses at mucosal sites and whether these mucosal epitope-specific CD8 + T-cell responses are clonally distinct from those seen in peripheral blood.

\section{RESULTS}

Comparable TCRB repertoires of epitope-specific CD8 + $T$ cells in prime-boost vaccinated and SIV-infected Mamu-A*01 + rhesus monkeys

We first sought to determine whether prime-boost vaccination strategies using different recombinant vectors induced clonally distinct virus-specific CD8 + T-cell responses. We did this by asking whether the epitope-specific CD $8+\mathrm{T}$-cell responses elicited by different vectors differed in their TCR repertoire or clonal diversity. We assessed the dominant SIV Gag p11C

${ }^{1}$ Division of Viral Pathogenesis, Department of Medicine, Beth Israel Deaconess Medical Center, Harvard Medical School, Boston, Massachusetts, USA.

Correspondence: P Sircar (piya.sircar@gmail.com)

Received 5 November 2011; accepted 26 April 2012; published online 4 July 2012. doi:10.1038/mi.2012.52 

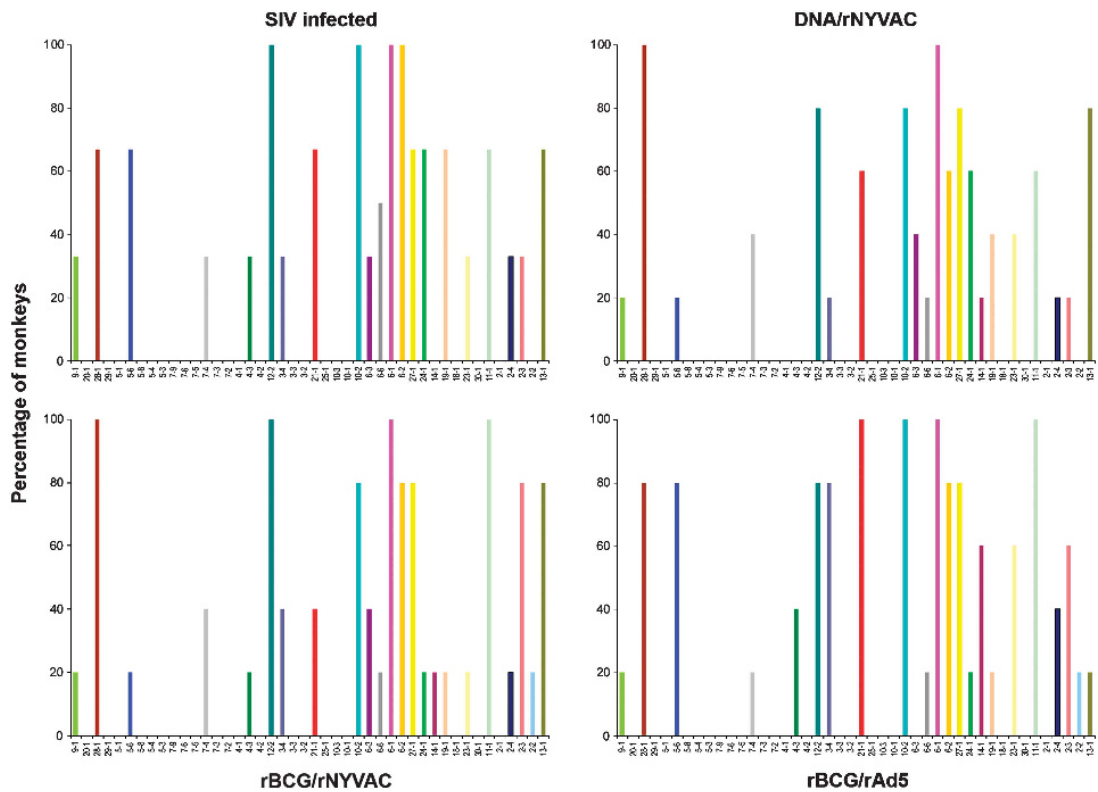

Figure 1 Comparable TCRBV gene usage by p11C-specific CD8 + T cells in prime-boost vaccinated and simian immunodeficiency virus (SIV)infected rhesus monkeys. The TCRBV gene family usage was determined in the peripheral blood p11C-specific CD $8+T$ cells of rhesus monkeys. TCRBV usage was compared in the three groups of prime-boost vaccinated rhesus monkeys (DNA/rNYVAC, rBCG/rNYVAC, and rBCG/rAd5) at 2 weeks following boost and a group of SIV-infected rhesus monkeys at 2 weeks following infection. The percentage of monkeys in each group expressing each TCRBV gene family is shown.

epitope-specific CD8 + T-cell response in the peripheral blood of the three groups of vaccinated, $M a m u-A^{*} 01+$ rhesus monkeys following immunization with either plasmid DNA prime/rNYVAC boost, rBCG prime/rNYVAC boost, or rBCG prime/rAd5 boost using Gag p11C/Mamu- $A^{\star} 01$ tetramer staining of peripheral blood cells. Each of these vectors carried a SIV Gag gene insert. Each vaccine group consisted of five monkeys. We also compared the p11C-specific CD8 + T-cell responses in these groups of vaccinated animals to those of a group of 6 SIVmac251-infected rhesus monkeys.

To determine whether there were any differences in the clonal repertoires of the SIV Gag-specific CD8 + T cells induced by prime-boost immunization and SIV infection, we examined the clonotypic composition of the p11C-specific CD8 + T-cell populations in these monkeys. We did this using a modified anchored template-switch PCR with reverse transcriptase to amplify, without bias, all expressed TCRB genes. The resulting PCR products were cloned and sequenced. Given the enormous variability in the TCR repertoires between individual monkeys, a large-scale analysis was needed to characterize the epitope-specific CD8 + TCR repertoires. Therefore, for each tissue compartment in every evaluated monkey, a minimum of 100 complete sequences were analyzed to define the representation of TCRB gene expression.

The TCRBV gene expression profile of these epitope-specific $\mathrm{CD} 8+\mathrm{T}$ cells was comparable in all the groups of vaccinated monkeys, with the TCRBV6 gene family expressed in all the groups (Figure 1). There were a total of 24 different TCRBV gene families that were expressed by the $\mathrm{p} 11 \mathrm{C}$-specific cell population in the three groups of vaccinated animals, and 19 of these TCRBV genes families were found in all the groups of animals. There was comparable diversity of TCRBV gene usage in all the groups of vaccinated monkeys, with each group expressing 21 or 23 TCRBV genes. Interestingly, the same dominant use of TCRBV6 and a comparable diversity of TCRBV usage were observed in the epitope-specific CD8 + T cells of the SIV-infected monkeys. Thus, prime-boost vaccination with different virus vectors and SIV infection induce p11C-specific CD8 + T-cell responses with comparable TCRBV gene usage and TCRBV diversity.

To complement this assessment of the p11C-specific CD8 + T-cell clonal repertoire, we also evaluated the TCRBJ gene usage and the CDR3 length. The relative representation of each TCRBJ family and the various CDR3 lengths were expressed as a percentage of the total clonotypes for each group of vaccinated and infected monkeys. We observed comparable representation of the different TCRJB gene families by the groups of vaccinated animals and SIV-infected animals (Figure 2a). A predominant representation of TCRJB1.5 was observed in the vaccinated and infected groups of monkeys. A CDR3 length of 15 amino acids was observed in at least $41 \%$ of the p11C-specific TCRB clonotypes in the SIV-infected and vaccinated monkeys (Figure 2b). Moreover, the representation of other CDR3 lengths was comparable in the vaccinated and infected monkeys. Additionally, we observed that inter- and intra-group variabilities in TCRBJ usage and CDR3 length were comparable. These comparisons of TCRBV, TCRBJ, and CDR3 length indicated that there was no particular skewing of the TCRB repertoires of the p11C-specific $\mathrm{CD} 8+\mathrm{T}$ cells generated in response to a particular immunization regimen, and these repertoires were comparable to those observed in SIV-infected monkeys. 

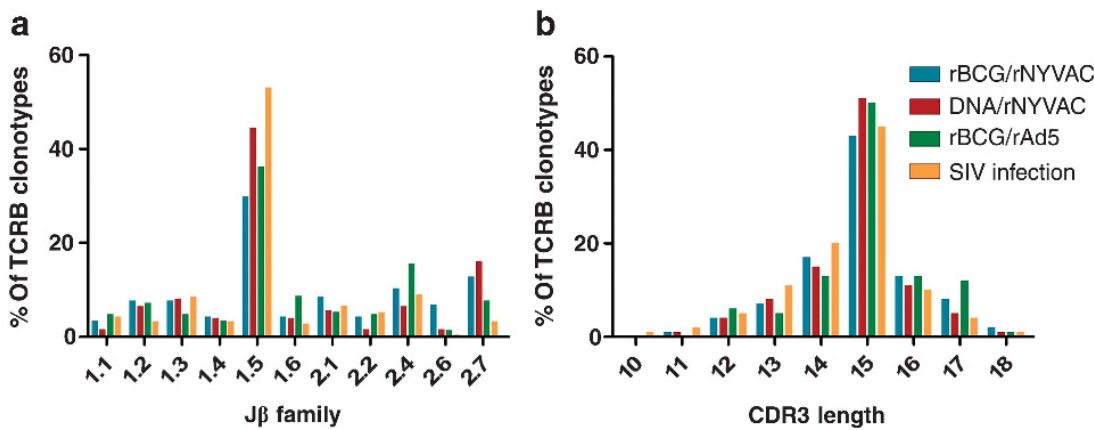

Figure 2 Comparable TCRBJ gene usage and CDR3 length in p11C-specific CD8 + T cells from prime-boost vaccinated and simian immunodeficiency virus (SIV)-infected rhesus monkeys. The TCRBJ gene family usage and CDR3 length were determined in the peripheral blood p11C-specific CD8 + T cells of rhesus monkeys. TCRBJ usage was compared in the three groups of prime-boost vaccinated rhesus monkeys (DNA/rNYVAC, rBCG/rNYVAC, and rBCG/rAd5) at 2 weeks following boost and a group of SIV-infected rhesus monkeys at 2 weeks following SIV infection. The p11C-specific clonotypes for all monkeys in each vaccinated or SIV-infected group were pooled to determine the proportion of p11C-specific clonotypes using (a) a particular TCRBJ gene and (b) having a particular CDR3 length.

Vaccination and SIV infection induced p11C-specific CD8 + T cells of comparable clonal diversity

To assess further whether different types of prime-boost vaccination regimens or SIV infection influence the clonal diversity of the epitope-specific CD8 + T-cell response, the clonal diversity was evaluated using the Simpson's diversity index (Figure 3). The Simpson's diversity index is a useful and accurate measurement of diversity of TCR repertoires that takes into account the frequencies of individual clonotypes and clonal dominance within a particular sample. ${ }^{11,14}$ The index varies in values between 0 and 1 , which reflect minimal and maximal diversity, respectively. The p11C-specific CD8 + TCR repertoire was very diverse 2 weeks following boost vaccination with the mean Simpson's diversity indices of approximately 0.9 for both the DNA/rNYVAC and rBCG/rNYVAC groups of monkeys. Thus, the p11C-specific CD8 + T-cell clonal repertoire upon boost was comparably broad in the DNA-primed and BCG-primed groups of vaccinated monkeys, suggesting that both vectors were comparable in priming virus-specific CD8 + $\mathrm{T}$ cells. The p11C-specific CD8 + TCR repertoire at 2 weeks following SIV infection had a trend towards even greater clonal diversity, with a mean diversity index of 0.96 .

The clonal diversities of the p11C-specific CD8 + T-cell populations were also evaluated at week 10 post boost and week 12 post infection to assess the evolution of these cellular immune responses. There was a statistically significant reduction in the clonal diversity of this cell population in both the groups of vaccinated animals by this later timepoint, with Simpson's diversity indices of $<0.85$. A similar reduction in clonal diversity was also observed in the SIV-infected animals, with a Simpson's diversity index of 0.83 in the peripheral blood sampled 12 weeks post infection. These studies indicate that the characteristics of the p11C-specific CD8 + T-cell clonal repertoire induced by different prime-boost immunization regimens and SIV infection are remarkably similar. Thus, the cognate antigen rather than

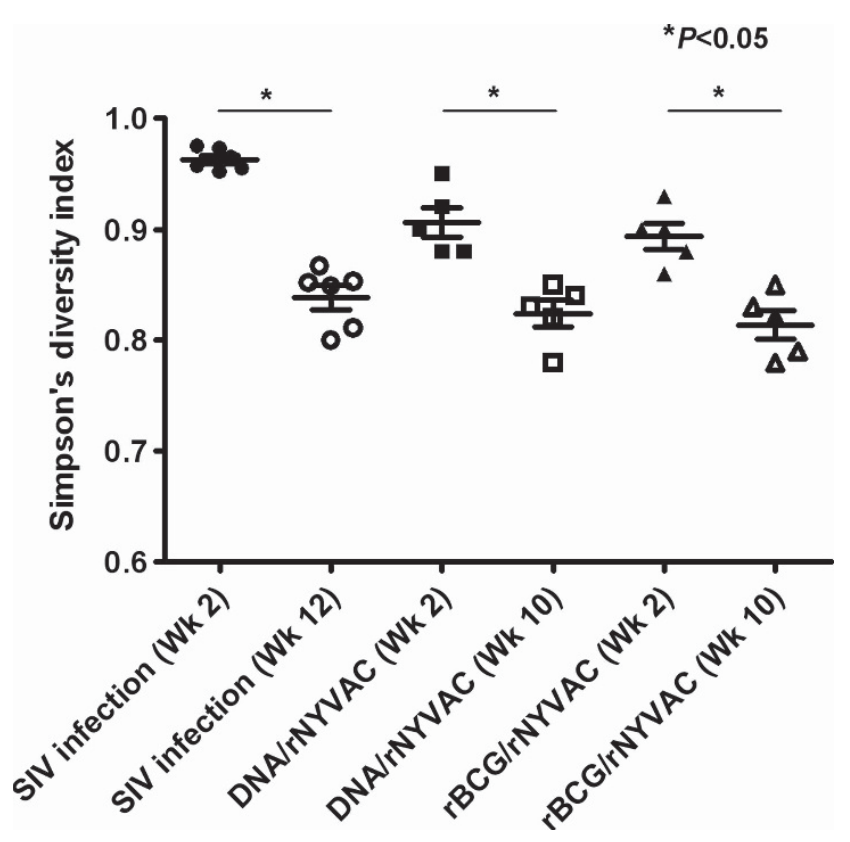

Figure 3 Prime-boost vaccination in rhesus monkeys induces clonally diverse $\mathrm{p} 11 \mathrm{C}$-specific CD8 + T-cell responses that undergo clonal focusing. The T-cell antigen receptor (TCR) repertoire of the p11C-specific CD8 + T cells in the peripheral blood of DNA/rNYVAC (squares) and rBCG/rNYVAC (triangles) vaccinated Mamu- $A^{*} 01$-positive rhesus monkeys was measured at 2 and 10 weeks (wks) (closed and open symbols, respectively) following boost. The TCR repertoire of p11C-specific CD8 + T cells in the simian immunodeficiency virus (SIV)infected rhesus monkey cohort is shown here for comparison (circles). Clonal diversity was assessed by calculating the Simpson's diversity index for each sample. The Simpson's index is a measurement of clonal diversity, where 0 denotes minimum diversity and 1 denotes maximum diversity. Means are shown for each group. There were statistically significant differences ( ${ }^{*} P$ values $<0.05$ using Welch's $t$ test) in cell populations sampled at week 2 and week 10 post boost in all the groups of vaccinated monkeys. 

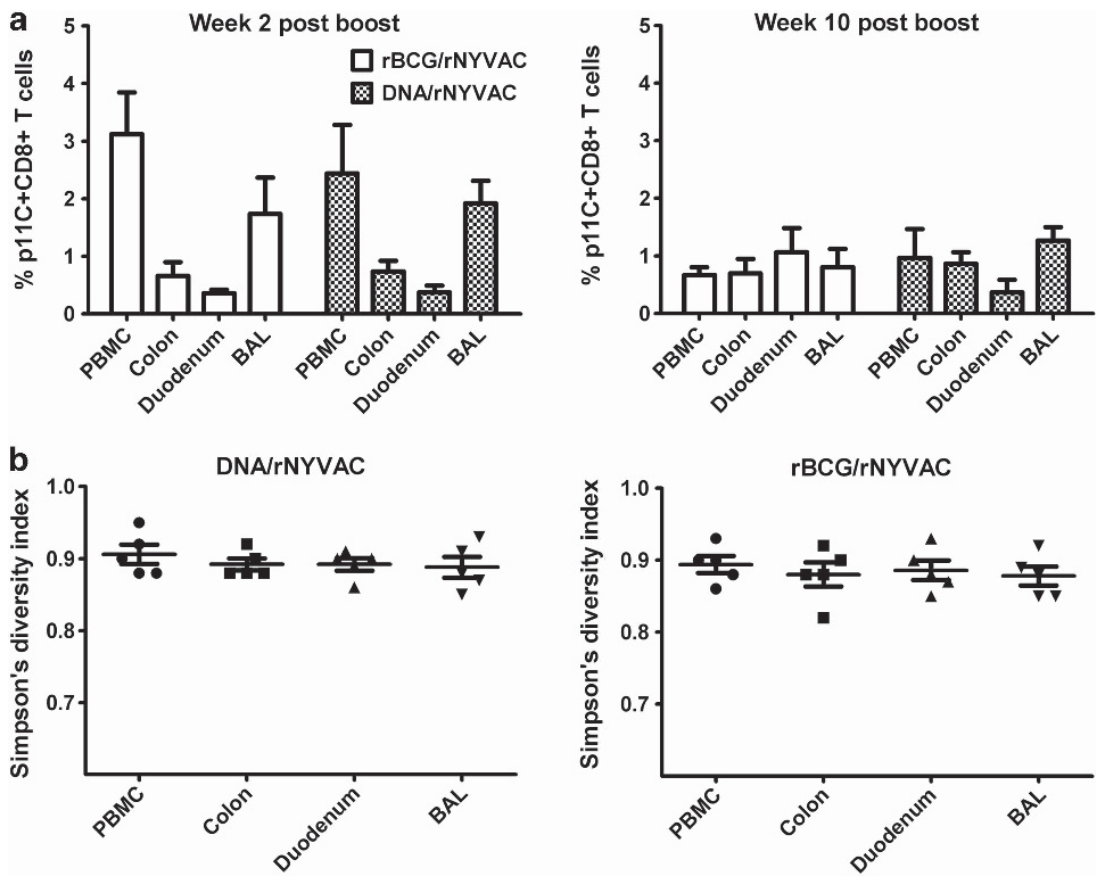

Figure 4 Systemic prime-boost vaccination with recombinant vectors induces clonally diverse p11C-specific CD8 + T-cell responses in mucosal compartments of rhesus monkeys. (a) The magnitude of Gag p11C-specific CD8 + T cell responses was evaluated in systemic and mucosal compartments of vaccinated Mamu- $A^{*} 01$-positive rhesus monkeys using Mamu- $A^{\star} 01 / p 11 \mathrm{C}$ tetramer staining. Cohorts of five rhesus monkeys vaccinated with either DNA/rNYVAC or rBCG/rNYVAC were evaluated at 2 and 10 weeks following rNYVAC boost. (b) Additionally, Simpson's diversity index was used to express the clonal diversity of the p11C-specific CD8+ T-cell responses in systemic and mucosal compartments of vaccinated monkeys at 2 weeks following rNYVAC boost. Means \pm s.e. for each group are shown. There were no significant differences in clonal diversity of the systemic and mucosal p11C-specific CD8 + T-cell populations. BAL, bronchoalveolar lavage; PBMC, peripheral blood mononuclear cells.

the presentation by a vaccine vector appears to be the primary determinant of clonotype recruitment in the blood.

\section{Systemic prime-boost immunization induces a sustained and clonally diverse dominant Gag epitope-specific mucosal CD8 + T-cell response in Mamu-A*01 + rhesus monkeys}

Next, we explored whether parenteral prime-boost vaccination with different recombinant vectors induces epitopespecific CD8 + T-cell responses in mucosal tissues. We assessed the dominant SIV Gag epitope-specific CD8 + T-cell response in the systemic and mucosal compartments following boosting of DNA/rNYVAC- and rBCG/rNYVAC-immunized Mamu- $A^{*}$ $01+$ rhesus monkeys using $\mathrm{p} 11 \mathrm{C} / \mathrm{Mamu}-\mathrm{A}^{*} 01$ tetramer staining of cells from the peripheral blood, gastrointestinal tissue (colon and duodenum), and bronchoalveolar lavage (BAL). Both the DNA/rNYVAC and rBCG/rNYVAC groups consisted of five monkeys each. No p11C-specific CD8 + T-cell response was detected in the peripheral blood or mucosal tissues of any of the animals 1 week following the boost immunization. The response in the blood was maximal at 2 weeks following boost, with a mean $3.1 \%$ of $\mathrm{CD} 8+\mathrm{T}$ cells being $\mathrm{p} 11 \mathrm{C}$-specific in the rBCG-primed group and $2.4 \%$ in the DNA-primed group; this response fell to 0.5 and $0.7 \%$, respectively, by week 10 following boost (Figure 4a). The kinetics of the response in BAL was comparable with that in the blood, with a peak response of $1.7 \%$ at week 2, falling to $0.6 \%$ at week 10 following boost in the rBCG-primed group and from 2 to $1.2 \%$ in the DNA-primed group. By contrast, the response in the gastrointestinal tissues was lower in magnitude than the response in the peripheral blood at week 2 , with tetramer responses $<0.8 \%$ in both the vaccinated groups of monkeys; by week 10 following the boosting immunization, the magnitude of the responses in the gastrointestinal tissues and peripheral blood were comparable.There were no significant differences observed in the magnitudes of the p11C-specific CD8 + T-cell responses induced by the DNA prime and $\mathrm{rBCG}$ prime in the peripheral blood and mucosal tissues at week 10 . These data indicate that systemic immunization with recombinant vectors induced the highest virus-specific CD8 + T-cell responses in the peripheral blood but also generated robust virus-specific CD8 + T-cell responses in mucosal sites. Furthermore, while the $\mathrm{CD} 8+\mathrm{T}$-cell response in the blood and BAL peaked rapidly and then waned, the CD8 + T-cell response in the GI tissues was sustained at a lower level.

Both DNA/rNYVAC and rBCG/rNYVAC immunization regimens induced clonally diverse p11C-specific CD $8+$ T-cell populations in the mucosal sites. At 2 weeks following rNYVAC boost, we observed Simpsons diversity indices $>0.88$ in the gastrointestinal mucosal tissues and BAL (Figure $4 \mathbf{b}$ ). There were no statistically significant differences between the clonal diversity of the $\mathrm{p} 11 \mathrm{C}$-specific $\mathrm{CD} 8+\mathrm{T}$-cell populations in the peripheral blood and in the mucosal tissues of the vaccinated animals, indicating that systemic vaccination does not restrict the clonal diversity of this cellular response in mucosal sites. 


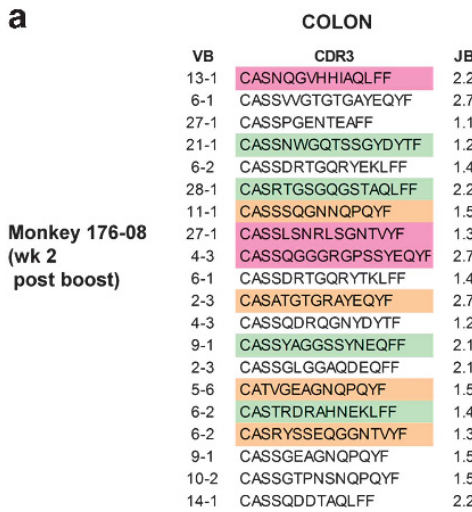

\begin{tabular}{cccll} 
& & \multicolumn{3}{c}{ DUODENUM } \\
B & count & VB & \multicolumn{1}{c}{ CDR3 } & JB \\
2 & 25 & $6-1$ & CASSITGTGPSTDPQYF & 1.5 \\
7 & 16 & $12-2$ & CASTGGSGEQFF & 2.1 \\
1 & 12 & $4-3$ & CASSQGGGRGPSSYEQYF & 2.7 \\
2 & 10 & $27-1$ & CASSLSNRLSGNTVF & 1.3 \\
4 & 9 & $6-2$ & CASSKESGTGRYEQYF & 2.7 \\
2 & 5 & $6-2$ & CASTRDRASNEKLFF & 1.4 \\
5 & 4 & $21-1$ & CASSAQEFYNSPLYF & 1.6 \\
3 & 4 & $13-1$ & CASNQGVHHIAQLFF & 2.2 \\
7 & 3 & $28-1$ & CASRTGSGQSTAQLFF & 2.2 \\
4 & 3 & $12-2$ & CASSPISRESYEQYF & 2.7 \\
7 & 2 & $6-2$ & CASSEFGNSNQPQYF & 1.5 \\
2 & 2 & $9-1$ & CASSYAGGSSYNEQFF & 2.1 \\
1 & 2 & $6-1$ & CASTRDRAHNEKLFF & 1.4 \\
1 & 1 & $6-1$ & CASSRDSSQETQYF & 2.4 \\
5 & 1 & $6-2$ & CASKKDRPSGEQYF & 2.7 \\
4 & 1 & $10-2$ & CASSNPDSGSQNTQYF & 2.4 \\
3 & 1 & $10-2$ & CASSEGNRADPQYF & 1.5 \\
5 & 1 & $10-2$ & CASSELAYEQYF & 2.7 \\
5 & 1 & $11-1$ & CASNSQGDNQPQYF & 1.5 \\
2 & 1 & $5-6$ & CATVGEAGNQPQYF & 1.5
\end{tabular}

\begin{tabular}{|c|c|c|}
\hline count & vB & $\begin{array}{l}\text { PBMC } \\
\text { CDR3 }\end{array}$ \\
\hline 19 & $27-1$ & CASSLSNRLSGNTVFF \\
\hline 13 & $7-4$ & CASSQAGTQGQNTQYF \\
\hline 11 & $11-1$ & CASSSQGNNQPQYF \\
\hline 10 & $10-2$ & CASSGTMNSNQPQYF \\
\hline 10 & $6-2$ & CASRYSSEQGGNTVFF \\
\hline 8 & $12-1$ & CASSSGTGVKTQYF \\
\hline 8 & $2-3$ & CASATGTGRAYEQYF \\
\hline 5 & $6-1$ & CASSEAPRGADTQYF \\
\hline 5 & $28-1$ & CASSNTLVSTDPQYF \\
\hline 3 & $21-1$ & CASSNWGQTSSGYDYTF \\
\hline 2 & 4-3 & CASSQGGGRGPSSYEQYF \\
\hline 2 & $6-1$ & CASSEARAAQNTQYF \\
\hline 1 & $6-1$ & CASSEARRSTDPQYF \\
\hline 1 & $6-2$ & CASSESRLTSGASVLTF \\
\hline 1 & $4-3$ & CASSQEDLKPQYF \\
\hline 1 & $6-2$ & CASSEFGNSNQPQYF \\
\hline 1 & $6-2$ & CASSKESGTGRYEQYF \\
\hline 1 & $10-2$ & CARNPGQGYEQYF \\
\hline 1 & $10-2$ & CASSELAYEQYF \\
\hline 1 & $11-1$ & CASNSQGDNQPQYF \\
\hline \multirow[t]{4}{*}{104} & $12-1$ & CASREQMNSPLYF \\
\hline & 13-1 & CASNQGVHHIAQLFF \\
\hline & $27-1$ & CASRDGDSLYNSPLYF \\
\hline & $27-1$ & CASSPNERNSGASUTF \\
\hline
\end{tabular}

$\begin{array}{cc}\text { JB } & \text { count } \\ 1.3 & 21 \\ 2.4 & 12 \\ 1.5 & 11 \\ 1.5 & 9 \\ 1.3 & 8 \\ 2.4 & 8 \\ 2.7 & 6 \\ 2.4 & 4 \\ 1.5 & 4 \\ 1.2 & 3 \\ 2.7 & 2 \\ 2.4 & 2 \\ 1.5 & 2 \\ 2.6 & 2 \\ 1.5 & 1 \\ 1.5 & 1 \\ 2.7 & 1 \\ 2.7 & 1 \\ 2.7 & 1 \\ 1.5 & 1 \\ 1.6 & 1 \\ 2.2 & 1 \\ 1.6 & 1 \\ 2.6 & 1 \\ & 104\end{array}$
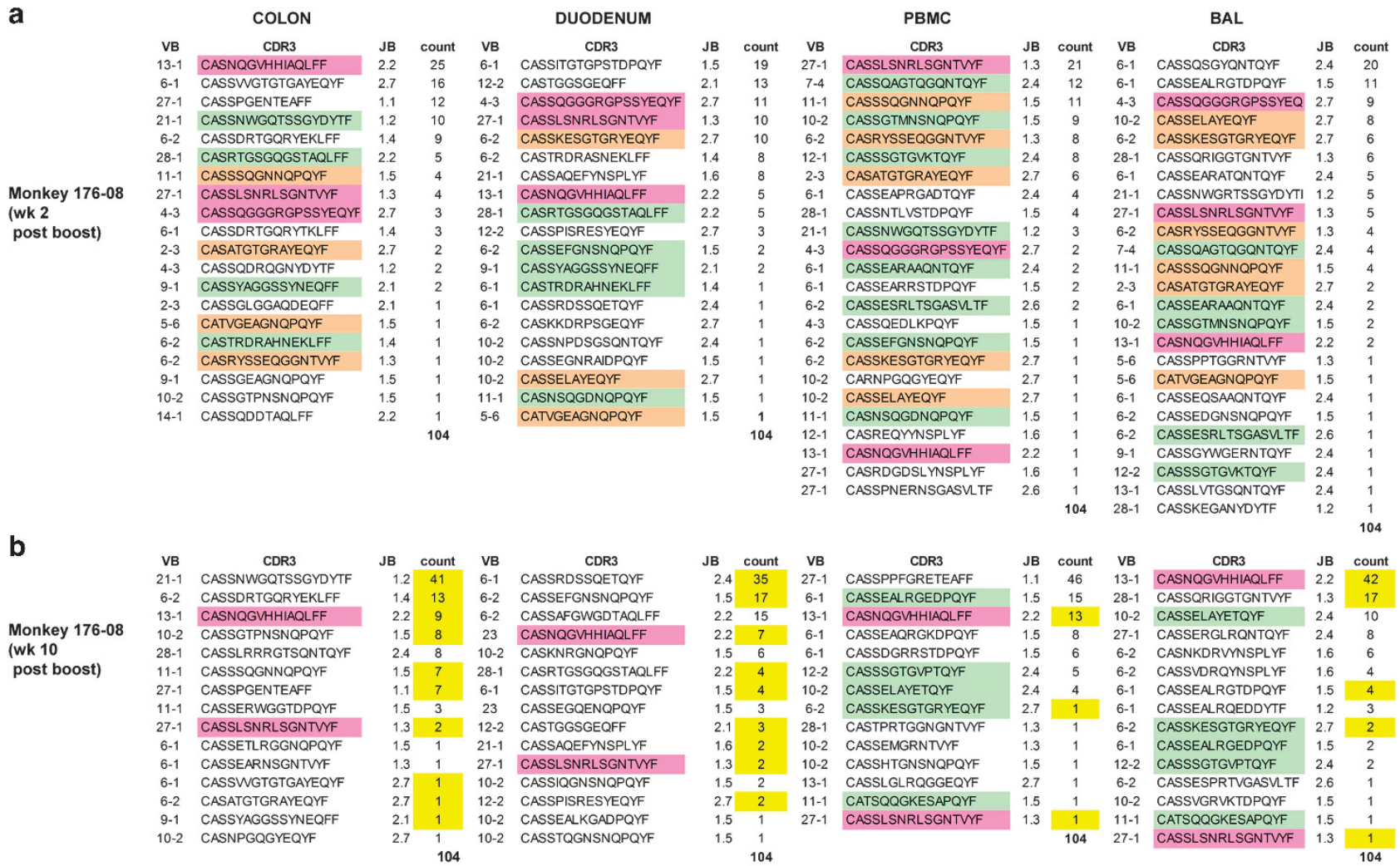

Found in

4 compartments

Also found at wk 2

Figure 5 Representation of clonotypes in p11C-specific CD8 + T-cell populations following prime-boost vaccination. TCRB CDR3 amino-acid sequences, TCRBV gene usage, TCRBJ gene usage, and the relative frequency of $\mathrm{p} 11 \mathrm{C}$-specific clonotypes are shown for representative monkey 176-08 at (a) week (wk) 2 and (b) week 10 following rNYVAC boost. Clones found in two compartments are shaded in green, clones found in three compartments are shaded in beige, and clones found in all evaluated compartments are shaded in pink. Yellow shading in the frequency column indicates that the specific clone was present in the same compartment at both week 2 and week 10 following boost. BAL, bronchoalveolar lavage; PBMC, peripheral blood mononuclear cells.

\section{The TCRB clonal repertoire of p11C-specific CD8 + T cells is shared between the mucosal tissues and peripheral blood early following vaccination but eventually diverges}

Having established the magnitude and kinetics of the p11C-specific CD8 + T-cell response in the mucosal tissues and peripheral blood, we then assessed the clonal composition of the p11Cspecific CD8 + T-cell responses in these anatomic compartments. For each tissue compartment in every evaluated animal, a minimum of 100 TCRB clones were completely sequenced to establish the clonotypic composition of the p11C-specific CD8 + T-cell population in each compartment. The amino-acid sequences spanning the CDR3 regions of the TCRB are shown for each anatomic compartment of the representative animal 176-08 at week 2 following boost (Figure 5a). Sharing of clonotypes between anatomic compartments of the same animal was defined by the presence of identical CDR3 nucleotide sequences in those compartments.

Analysis of the TCRB gene usage in the p11C-specific CD8 + T-cell population derived from the peripheral blood at week 2 following boost demonstrated a polyclonal repertoire, with 24 clonotypes seen in 104 sequences. At week 2 following boost, the p11C-specific CD8 + T cells from the colon, duodenum, and BAL exhibited similar polyclonality, with 20,20 , and 25 individual clonotypes defined, respectively. The clonal repertoires in these compartments were very similar, and $>50 \%$ of the clones in each compartment were also found in other compartments. There were three clones, highlighted in pink, present in all four compartments that were studied. There were also 11 clones shared in two compartments, highlighted in green, and 6 clones shared in three compartments, highlighted in beige. A high degree of intercompartmental sharing was observed for both the dominant and the rare clones.

Next, we characterized the p11C-specific CD8 + T-cell clonal repertoire at week 10 post boost to determine how the repertoire evolved (Figure 5b). The repertoire diversity decreased by week 10 in the peripheral blood and the mucosal compartments in this monkey, with fewer than 15 distinct clones defined from 104 total sequences.

In contrast to what was observed early post boost, the dominant p11C-specific CD8 + T-cell clones in the colon and duodenum at week 10 were unique to those anatomic compartments, whereas the clones in the peripheral blood and BAL were shared. There was a significant decrease in the sharing of clones between the gastrointestinal mucosal compartments 

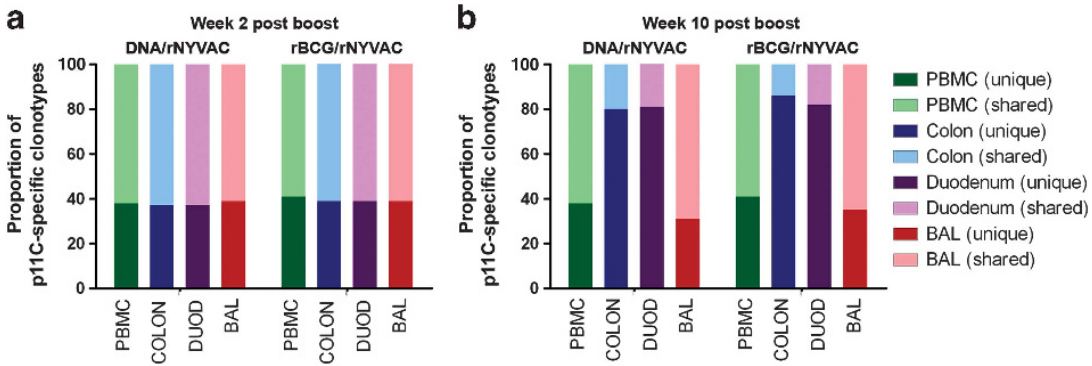

Figure 6 Longitudinal analysis of clonotypic sharing in mucosal and systemic compartments of vaccinated rhesus monkeys. The mean percentages of p11C-specific CD8 + T-cell clonotypes detected in only one (unique) or more than one (shared) compartments are shown for four anatomic compartments (peripheral blood mononuclear cells (PBMC), bronchoalveolar lavage (BAL), colon, and duodenum (DUOD)) in the entire cohort of DNA/rNYVAC- and rBCG/rNYVAC-vaccinated rhesus monkeys at (a) week 2 and (b) week 10 following boost. The total numbers of clonotypes in each individual compartment were pooled in each cohort of vaccinated monkeys to generate these values.

and the peripheral blood and BAL by week 10 post boost, as indicated by the large number of unshared sequences $(>80 \%$ in the colon and duodenum). By contrast, $>50 \%$ of clones were shared between the peripheral blood and BAL at week 10, similar to what was observed at 2 weeks following the boost immunization. Additionally, we observed that clones were retained in the colon and duodenum to a much greater degree than in the peripheral blood or BAL (highlighted in yellow).

We observed substantial sharing of p11C-specific CD8 + $\mathrm{T}$-cell clones between the systemic and mucosal compartments at 2 weeks following boosting in the groups of DNA/rNYVAC- and rBCG/rNYVAC-vaccinated monkeys (Figure 6a). Greater than $60 \%$ of the clones in an anatomic compartment were also found in one or more other compartments in the same animal; only a minority of the clones were present in a single compartment. However, by 10 weeks following boosting, there was a substantial decrease in clonal sharing between the gastrointestinal mucosal compartments and peripheral blood (Figure $\mathbf{6 b}$ ). In the DNA/rNYVAC group of vaccinated monkeys, the number of unique clonotypes in the colon and duodenum increased to $78 \%$ of the total number of clones detected in each of these compartments. Similarly, in the $\mathrm{rBCG} / \mathrm{rNYVAC}$ group, $>80 \%$ of the total clones in the colon and duodenum were unique to these compartments. Interestingly, we did not observe a decrease in clonal sharing between the peripheral blood and BAL in the vaccinated monkeys. Greater than $60 \%$ of the clones were found in both compartments, and there was no significant change in clonal sharing between these compartments over time. Additionally, even if the colon and duodenum are considered as a single mucosal compartment, we observed the same trend, with a majority of the clones in the gastrointestinal mucosa unique to that compartment, whereas there were a small number of clones that were shared between the gastrointestinal mucosa, the peripheral blood and BAL.

\section{Vaccine-induced p11C-specific CD8 + T-cell clones were retained in the gastrointestinal mucosal compartments}

To determine what was driving the decrease in clonal sharing between the gastrointestinal mucosal compartments and other anatomic compartments, we explored whether there were differences in the retention and turnover of p11C-specific CD8 + T-cell clones in these compartments (Figure 7). We found that approximately $80 \%$ of the clones in the colon and duodenum of the vaccinated animals were retained from week 2 to week 10 following boost, while $20 \%$ of clones were only found at the week 10 timepoint. By contrast, there was a more equal distribution of clones retained in the peripheral blood and BAL of the vaccinated animals during this time period, with approximately $50 \%$ of the clones retained in the peripheral blood and 58\% of clones retained in the BAL. In SIV-infected animals, there were comparable percentages of $\mathrm{p} 11 \mathrm{C}$-specific CD8 + T-cell clones retained at weeks 2 and 10 following infection, and this was observed in all of the evaluated anatomic compartments.

\section{Dynamic expression of trafficking molecules on vaccine-induced $p 11 C$-specific $C D 8+T$ cells}

The sharing of p11C-specific CD8 + T-cell clones between systemic and mucosal compartments found soon after immunization followed by the decrease in this sharing suggested that there may be changes in the ability of the peripheral blood p11Cspecific CD8 + T cells to traffic to the gastrointestinal mucosa. To explore this possibility, we examined the dynamics of $\alpha 4 \beta 7$ expression on p11C-specific CD8 + T cells in the peripheral blood following vaccination. The gating strategy used and representative staining profiles for $\beta 7$ are shown in Supplementary Figure S2 online. We observed high levels of $\alpha 4 \beta 7$ expression at week 2 with $50 \%$ of vaccine-induced p11C-specific CD $8+\mathrm{T}$ cells expressing this molecule following boosting (Figure 8a). However, there was a decrease to $7 \%$ of cells expressing $\alpha 4 \beta 7$ by week 10 following boost. In contrast, we did not observe a significant decrease in $\alpha 4 \beta 7$ expression on $\mathrm{p} 11 \mathrm{C}$-specific CD8 + $\mathrm{T}$ cells between week 2 and week 12 following infection. These findings suggested that the limited duration of $\alpha 4 \beta 7$ expression on the vaccine-induced p11C-specific $\mathrm{CD} 8+\mathrm{T}$ cells may explain the transient ability of these cells to traffic to the gastrointestinal mucosa. The relatively small proportion of new $\mathrm{p} 11 \mathrm{C}$-specific CD8 + T-cell clones found in the gastrointestinal mucosal compartments at week 10 following boost might be explained by 

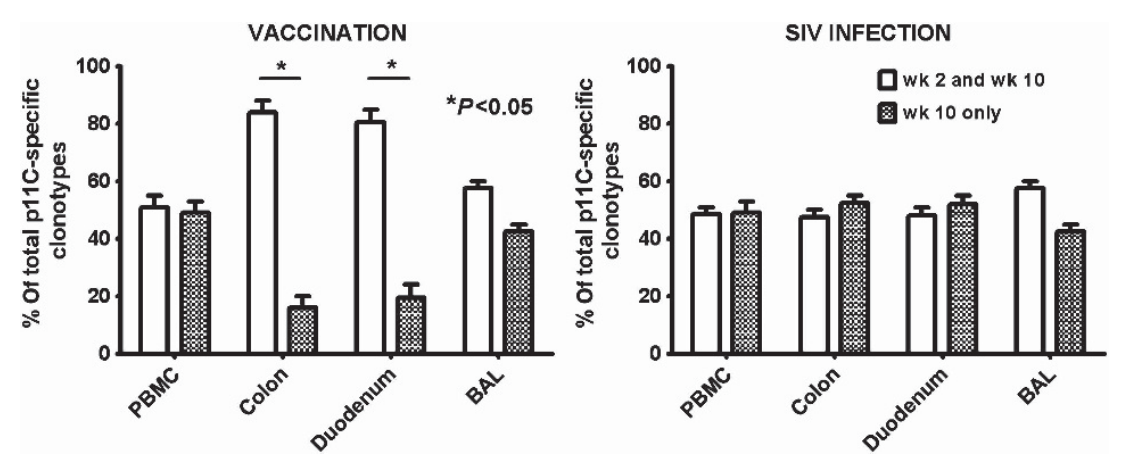

Figure 7 Vaccine-induced p11C-specific CD8 + T-cell clones are retained in the gastrointestinal mucosa. The percentage of retained and new p11C-specific CD8 + T-cell clones are shown for four anatomic compartments in vaccinated rhesus monkeys at 10 weeks (wks) following rNYVAC boost and in simian immunodeficiency virus (SIV)-infected rhesus monkeys 12 weeks following infection. Retained clones are those found in the same compartment at 2 weeks and 10 weeks following rNYVAC boost, whereas new clones are those identified only 10 weeks following this boost. The total numbers of clonotypes in each individual compartment were pooled for each cohort of DNA/rNYVAC- and rBCG/rNYVAC-vaccinated animals. Means \pm s.e. for each compartment are shown. There were statistically significant differences $(P<0.05$, Welch's $t$ test $)$ between the percentages of retained clones and new clones in the gastrointestinal mucosal compartments of the vaccinated monkeys. BAL, bronchoalveolar lavage; PBMC, peripheral blood mononuclear cells.
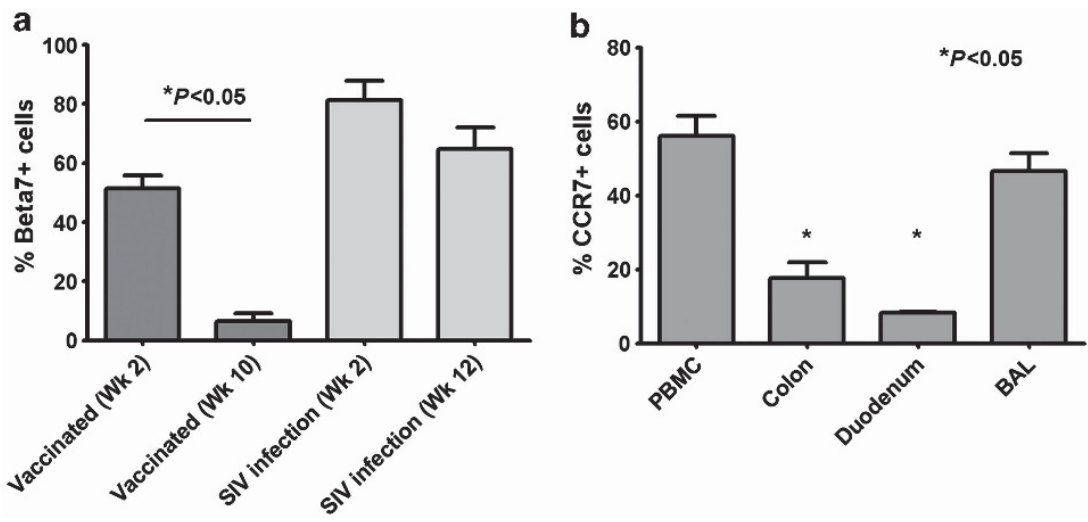

Figure 8 Dynamic expression of molecules associated with cell trafficking on vaccine-induced p11C-specific CD8 + T cells. (a) The percentages of the peripheral blood p11C-specific CD $+\mathrm{T}$ cells expressing the gastrointestinal-homing-associated cell surface molecule $\alpha 4 \beta 7$ are shown at 2 and 10 weeks (wks) following rNYVAC boost in vaccinated animals and at 2 and 12 weeks following simian immunodeficiency virus (SIV) infection. Means \pm S.e. for each group at each timepoint are shown. There was a statistically significant decrease in $\alpha 4 \beta 7$ expression over time in vaccinated animals ( $P<0.05$, Welch's $t$ test). (b) The percentages of p11C-specific CD8 + T cells expressing CCR7 in different anatomic compartments of vaccinated animals were measured at week 2 following rNYVAC boost. Means \pm s.e. for each compartment are shown. There were statistically significantly lower levels of CCR7 expression on the gastrointestinal mucosal p11C-specific CD8 + T cells as compared with the peripheral blood and bronchoalveolar lavage (BAL) $(P<0.05$, Kruskal-Wallis test). PBMC, peripheral blood mononuclear cells.

the reduction in the gastrointestinal-homing ability of vaccineinduced $\mathrm{p} 11 \mathrm{C}$-specific CD8 $+\mathrm{T}$ cells over time.

We also evaluated the expression of CCR7, the chemokine receptor which is important for lymphocyte circulation in secondary lymphoid organs, on vaccine-induced $\mathrm{p} 11 \mathrm{C}$-specific CD8 + T cells. Although $>50 \%$ of the p11C-specific CD $8+\mathrm{T}$ cells in the peripheral blood and BAL of the vaccinated animals expressed CCR7, $<10 \%$ of p11C-specific CD $8+$ T cells in the gastrointestinal mucosal compartments expressed CCR7 (Figure 8b). The downregulation of CCR7 expression by these cells in the gastrointestinal mucosal tissue is consistent with the high level of retention of the vaccine-induced p11C-specific CD8 + T-cell clones in this anatomic compartment late following boosting.

\section{Gastrointestinal mucosal p11C-specific cells have an effector memory phenotype}

We also examined the memory differentiation profile of the vaccine-induced $\mathrm{p} 11 \mathrm{C}$-specific $\mathrm{CD} 8+\mathrm{T}$ cells in mucosal and systemic compartments. We used memory subset designations based on the expression of CD28 and CCR7 to determine the distribution of central memory $(\mathrm{CD} 28+/ \mathrm{CCR} 7+)$, transitional effector memory (CD28 +/CCR7 -$)$, and fully differentiated effector memory (CD28 - /CCR7 - ) p11C-specific CD8 + T cells in these compartments after rNYVAC boost. ${ }^{3}$ The gating strategy used and representative staining profiles for CD28 and CCR7 are shown in Supplementary Figure S2 online. At week 2 following boost, $>50 \%$ of the p11C-specific CD $8+\mathrm{T}$ cells in the peripheral blood had a central memory phenotype, 

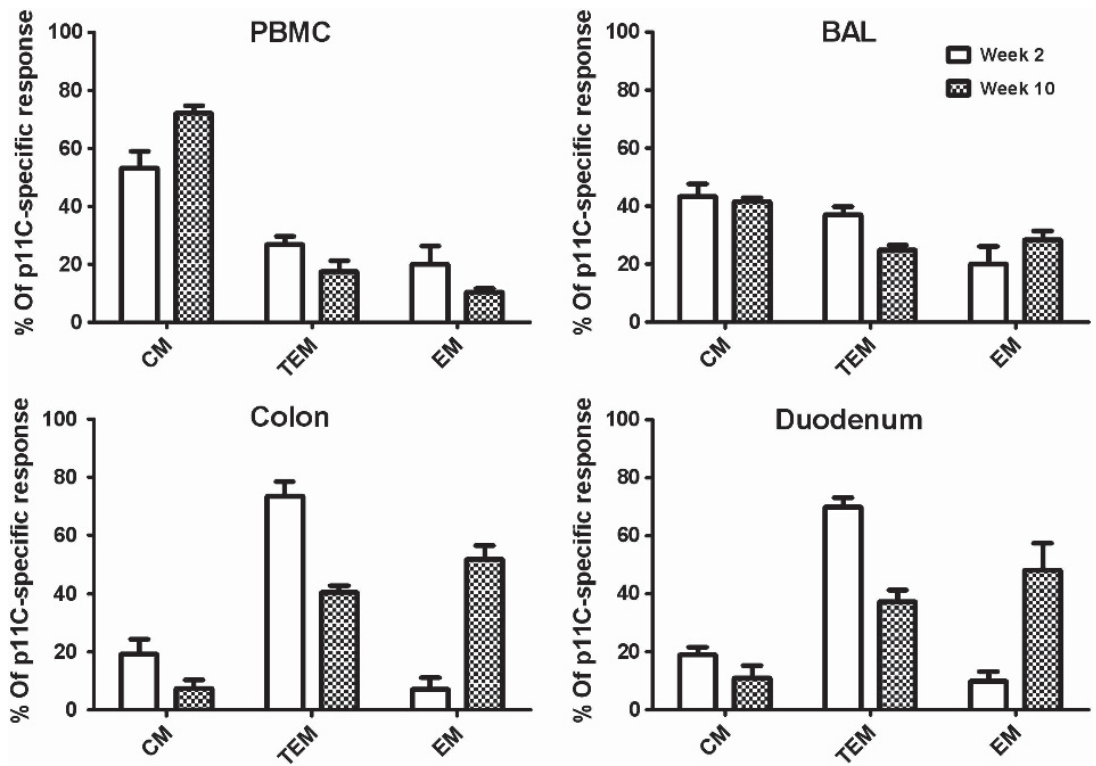

Figure 9 Systemic prime-boost vaccination induces effector memory p11C-specific CD8 + T cells in the gastrointestinal mucosa. The memory subsets of vaccine-elicited p11C-specific CD $8+T$ cells in various anatomic compartments were determined by analysis of the expression of the cell surface molecules CD28 and CCR7. The relative representation of the p11C-specific CD8 + T cells with a central memory $(C M)(C D 28+/ C C R 7+)$, a transitional effector memory (TEM; CD28+/CCR7-), and a fully differentiated effector memory (EM) phenotype (CD28-/CCR7-) are shown at 2 and 10 weeks following rNYVAC boost. BAL, bronchoalveolar lavage; PBMC, peripheral blood mononuclear cells.

and $>40 \%$ had this phenotype in the BAL (Figure 9). However, in both the colon and duodenum, $>70 \%$ of the p11C-specific CD8 + T-cell response had an effector memory phenotype. The majority of the effector memory p11C-specific CD $8+\mathrm{T}$ cells in the gastrointestinal mucosal tissue were transitional effector memory cells. At week 10 following boost, $70 \%$ of these cells in the peripheral blood had a central memory phenotype, and there was a decrease in the percentage of cells with an effector memory phenotype. There were no significant changes over time in the memory profile of these cells in the BAL. However, in the gastrointestinal mucosal tissue, we observed a relative decrease of the transitional effector memory population, with an associated increase in the proportion of p11C-specific CD8 + $\mathrm{T}$ cells with a fully differentiated effector memory phenotype. Thus, the microenvironment of the gastrointestinal mucosal tissue affects memory differentiation of these SIV Gag epitopespecific CD8 + T cells.

\section{DISCUSSION}

In this study, we showed that different prime-boost vaccination regimens (rBCG/rNYVAC, rDNA/rNYVAC, and rBCG/rAd5) generate SIV-specific clonal responses with comparable diversity. We observed that epitope-specific CD $8+\mathrm{T}$ cells elicited by the three different prime-boost vaccination regimens had similar TCRBV usage, TCRBJ usage, and CDR length. Moreover, the vaccine-elicited epitope-specific CD8 + T-cell responses were as clonally diverse as those induced by SIV infection. This was surprising, as one might expect a persistently replicating virus to generate a greater breadth of clonal diversity than vaccination with non-replicating vectors. These results suggest that although the nature of the vaccine vector might influence the magnitude of the CD8 + T-cell responses, the diversity and clonal composition of the CD8 + T-cell repertoire cannot be manipulated by different vectors. Our data support previous studies of the p11Cspecific CD8 + T-cell repertoire that have also found similar clonal composition of the p11C-specific response during SIV infection and vaccination. ${ }^{15}$

An important question for HIV vaccine design is whether systemic vaccination can induce potent and durable cellular responses at the mucosal sites. We have utilized the well-studied p11C epitope of SIV Gag to better understand the effects of systemic vaccination in inducing mucosal CD $8+$ T-cell responses. ${ }^{9,15,16}$ It has been assumed that systemic vaccination might not be optimal because of compartmentalization between the systemic and mucosal immune systems. In fact, several studies of localized infection and vaccination have shown anatomic skewing of primary and memory CD8 + T-cell responses to the initial site of antigen exposure. ${ }^{1,2,17-19}$ There is evidence to suggest that tissue-homing receptor expression is determined by the location of T-cell activation, such that priming in the mesenteric lymph node induces gastrointestinal-homing $\alpha 4 \beta 7$ expression. ${ }^{20,21}$ These observations suggest that local infection/ immunization and subsequent priming in the mucosal tissues may be required for dissemination of $\mathrm{CD} 8+\mathrm{T}$ cells into the gastrointestinal and genital mucosal sites. ${ }^{2,17,22,23}$ However, the presented data suggest that systemic vaccination with non-replicating vectors induces rapid and clonally diverse mucosal cellular immune responses that are sustained over time. Other recent studies of systemic vaccination as well as localized infection have also demonstrated similar broad effector T-cell responses that bridge systemic and mucosal compartments. ${ }^{6,24,25} \mathrm{An}$ explanation consistent with these findings is that regulation of 
T-cell trafficking is dynamically modulated upon trafficking to different microenvironments. For example, there is evidence showing that priming in the spleen can generate a promiscuous homing receptor program that drives migration to the gastrointestinal mucosa. ${ }^{26}$ Similarly, intradermal inoculation of vaccinia virus can result in upregulation of gastrointestinalhoming molecules. ${ }^{27}$

Another issue of relevance to vaccine-induced protective cellular immunity is whether memory CD8 $+\mathrm{T}$ cells circulate between the blood and the mucosal tissues. Parabiosis experiments and adoptive transfer studies in mice have suggested that there is restricted recirculation of CD8 + T cells between the blood and the intestinal epithelium, whereas CD8 + T cells can recirculate between the blood and the lungs. ${ }^{28,29}$ In the current studies, at week 2 following vaccination there was a high degree of clonal sharing between all sampled anatomic compartments; however, by week 10 following vaccination there were very few clones shared between the peripheral blood and the gastrointestinal mucosal tissue. A large proportion of p11C-specific CD8 + $\mathrm{T}$ clones were retained in the gastrointestinal mucosa, whereas there was turnover of these clones in the peripheral blood and BAL. Consistent with these observations, we found small numbers of CCR7 + p11C-specific CD8 + T cells in mucosal sites, as CCR7 is involved in the exit of CD8 + T cells from the tissues into the afferent lymphatics. ${ }^{30,31}$ The limited clonal sharing of p11C-specific CD8 + T cells between the peripheral blood and the gastrointestinal mucosal tissues might be a consequence of the low level of expression of $\alpha 4 \beta 7$ on p11C-specific CD8 + $\mathrm{T}$ cells in the peripheral blood following vaccination. In fact, the present data are consistent with a study that demonstrated a dynamic CD8 + T-cell migration program, with transient expression of $\alpha 4 \beta 7$ on these cells following LCMV infection in mice and yellow fever vaccination in humans. ${ }^{26}$ Additionally, we found that the percentage of p11C-specific CD $8+\mathrm{T}$ cells remained stable in the gastrointestinal mucosal tissues from week 2 to week 10 following vaccination even though there was a decrease in $\alpha 4 \beta 7$ expression by this cell population in the peripheral blood. This finding suggests that there are mechanisms in addition to trafficking that ensure the maintenance of the p11C-specific CD8 + T-cell population in the mucosa. The available studies suggest that vaccine-induced CD8 $+\mathrm{T}$ cells migrate into the intestinal mucosa, differentiate into effector memory CD8 + T cells in situ, and remain resident there without recirculating. In the future, it will be important to evaluate what specific mechanisms (in situ proliferation or resistance to cell death) allow the maintenance of resident effector memory $\mathrm{CD} 8+\mathrm{T}$ cells in the mucosal tissues.

The absence of sharing of p11C-specific CD8 + T-cell clones between mucosal and systemic compartments at a late timepoint following vaccination contrasts with our previous demonstration of increased clonal sharing in these compartments over time following SIV infection. This difference suggests that there may be differences in the $\mathrm{CD} 8+\mathrm{T}$-cell migration program induced by SIV infection and vaccination, perhaps owing to the effect of sustained antigen expression on the migration and activation of $\mathrm{CD} 8+\mathrm{T}$ cells. ${ }^{32}$ Thus, transient antigen expression following vaccination with non-replicating vectors may result in limited trafficking of CD8 + T cells to the gastrointestinal mucosa, whereas chronic antigenic stimulation in the setting of SIV infection in the blood and gastrointestinal mucosa may allow the responding memory CD $8+\mathrm{T}$ cells to traffic between these compartments.

In contrast to what we observed in the gastrointestinal mucosal tissues, we demonstrated a high degree of sharing of p11C-specific CD8 + T-cell clones between the peripheral blood and BAL at both the early and late timepoints following vaccination, and detected very few clones retained in the BAL over time. These findings are consistent with the previous observations that Sendai virus-specific CD8 $+\mathrm{T}$ cells recirculate between pulmonary airways and the periphery. ${ }^{33}$

It has been suggested that the differentiation state of vaccineinduced memory CD8 $+\mathrm{T}$ cells is important in the protection that these cells confer. Effector memory CD8 + populations with constitutive cytolytic function may be more beneficial in controlling virus replication than central memory CD8 + T-cell populations. It has recently been shown that live, replicating vaccine vectors such as rhesus monkey cytomegalovirus induce differentiated effector memory CD8 + T-cell responses that may confer protection from mucosal SIV challenge. ${ }^{3,34}$ In the present studies, we have evaluated non-persistent, nonreplicating vaccine vectors that have been shown to induce central memory CD8 $+\mathrm{T}$ cells. However, it is noteworthy that there was a predominance of central memory CD8 $+\mathrm{T}$ cells in the peripheral blood and BAL, but a predominance of effector memory CD8 $+\mathrm{T}$ cells in the gastrointestinal mucosa of these vaccinated animals. We also observed an evolution of p11C-specific CD8 + T cells following vaccination from the transitional effector memory phenotype to effector memory phenotype in the gastrointestinal mucosal tissue. These findings suggest that the memory phenotype of $\mathrm{CD} 8+\mathrm{T}$ cells in mucosal tissues may be independent of the vaccination strategy. Regardless of initial stimulation, memory CD8 $+\mathrm{T}$ cells in the gastrointestinal mucosa may evolve to an effector differentiation state, as a consequence of exposure to local environmental factors such as commensal microorganisms and the tissue-associated cytokine milieu. ${ }^{28}$ In fact, memory CD8 + T cells in the gastrointestinal epithelium are functionally distinct from those in lymphoid tissue and mediate immediate cytotoxicity upon reinfection. ${ }^{35}$ Although it is unclear how tissue microenvironments influence memory differentiation, the present data as well as the other studies suggest that anatomic location can impact the memory CD8 + T-cell differentiation. ${ }^{28,35,36}$ In the present study, due to the extremely limited number of $\mathrm{p} 11 \mathrm{C}+\mathrm{CD} 8+\mathrm{T}$ cells obtained from mucosal pinch biopsies, we were unable to carry out functional studies on these cells such as intracellular cytokine staining or proliferation assays. In future studies, it might be helpful to obtain necropsy samples from vaccinated and infected rhesus monkeys to provide enough cells to carry out functional assays.

Although the present studies focused on the gastrointestinal mucosal tissues, which are both an early and long-term reservoir of viral replication, it is important to note that the genital 
mucosa is the first point of virus acquisition in heterosexual HIV-1 transmission. Our focus on gastrointestinal mucosal tissues reflects, in part, the substantial difficulties associated with sampling the genital mucosal tissue in rhesus monkeys. Given our finding that individual mucosal tissues are associated with a distinct profile of CD8 + T-cell responses, it is likely there are unique features of the genital mucosa that should be considered in future studies.

The present study demonstrates that systemic vaccination can elicit CD8 + T-cell responses in mucosal compartments. However, it also shows that there may be vaccine-related differences in the trafficking, phenotype, and function of CD8 + T cells at mucosal sites that can impact the protective ability of vaccineinduced immune responses. This observation may have important implications. First, the character of virus-specific CD8 + $\mathrm{T}$ cells in the peripheral blood may not reflect the quantity and quality of nonrecirculating mucosal cell populations. Second, the observation that virus-specific memory CD8 + T cells can remain resident in the gastrointestinal mucosal tissue supports the hypothesis that microenvironmental cues can influence the differentiation profile of these cells. Thus, evaluating correlates for vaccine-induced immunity for HIV/SIV will require the study of genital and gastrointestinal mucosal cell populations.

\section{METHODS}

Animal immunizations and sampling. All animals used in this study were colony-bred $M a m u-A^{\star} 01+$ Indian-origin rhesus monkeys (Macaca mulatta). The animals were maintained in accordance with the guidelines of the Institutional Animal Care and Use Committee for Harvard Medical School. There were three groups of systemically prime-boost immunized monkeys and one group of SIV-infected monkeys used in these studies. The groups were primed with DNA $(5 \mathrm{mg}$, intramuscular, at 0,4 , and 8 weeks $)$ or $\mathrm{rBCG}\left(10^{9} \mathrm{cfu}\right.$, intravenous $)$ vectors expressing the SIVmac239 Gag gene. These animals were then boosted with an attenuated recombinant poxvirus vector (rNYVAC, $10^{7} \mathrm{pfu}$, intramuscular, at 43 weeks) or a recombinant adenovirus serotype 5 vector (rAd5, $10^{10} \mathrm{pfu}$, intramuscular, at 43 weeks) expressing the same gene. Each vaccinated group consisted of five monkeys. The fourth group consisted of six monkeys infected with SIVmac251 as previously described. ${ }^{13}$ We obtained peripheral blood, colon biopsy, duodenal biopsy, and BAL specimens from all the animals.

Sample preparation and flow cytometric sorting. Lymphocytes were isolated from the blood and BAL by using Ficoll-Paque (Pharmacia, NY). Isolation of mononuclear cells from colonic and duodenal biopsies was accomplished by using an optimized collagenase digestion procedure for $30 \mathrm{~min} .{ }^{37}$ The tetrameric Mamu-A*01/p11C complex was used in association with CD3 (SP34.2)-, CD8 (SK1)-, CD28 (28.2)-, CD95 (DX2)-, CCR7 (3D12)-, and $\beta 7$ (FIB504)-specific antibodies to stain lymphocytes and identify p11C + CD $8+T$ cells from the peripheral blood and mucosal compartments. A violet fluorescent reactive dye (ViViD; Invitrogen, Carlsbad, CA) was also used as a viability marker to exclude dead cells. The antibodies used in the present study were directly coupled to FITC, PE, ECD, PE-Cy7, APC, APC-Cy7, and Quantum-Dot 605. All antibodies were purchased from BD Biosciences (San Jose, CA). Sorting of viable, p11C tetramer $+\mathrm{CD} 3+\mathrm{CD} 8+\mathrm{T}$ cells was performed using a BD FACSAria sorter.

Clonotype analysis. Up to 20,000 p11C tetramer-labeled CD8 + T cells were sorted directly into 96-well plates containing $25 \mu$ lof lysis buffer from the Total Mammalian RNA Extraction Kit (Sigma, St Louis, MO).
The purity of the p11C-specific CD $8+\mathrm{T}$ cells was $>97 \%$, and representative staining profiles and post-purity plots demonstrating this purity are shown in Supplementary Figure $\mathbf{S} 1$ online. Comparable numbers of p11C-specific CD8 + T cells were sorted from the various compartments of each monkey, and a summary of the number of p11C-specific CD8 + T cells obtained from each monkey is shown in Supplementary Table S1 online. The p11C-specific CD8 + T-cell population from each compartment was sorted into a separate well of a 96-well plate to prevent cross-contamination. Cell samples were then centrifuged for $3 \mathrm{~min}$ and immediately stored at $-80^{\circ} \mathrm{C}$. Subsequently, cell samples were thawed and RNA was extracted using the Total Mammalian RNA Extraction Kit (Sigma). cDNA synthesis from the resulting RNA was conducted with the 5' SMART RACE cDNA amplification kit (Clontech, Mountain View, CA). The cDNA was subjected to template switch-anchored PCR with reverse transcriptase for unbiased amplification of the TCRB chain gene products using a universal primer mix and a $C \beta R$ primer (rhesus monkey TCRBC specific; GATCTCTGCTTCTGATGGCTCAA) as previously described. ${ }^{13}$ Purified PCR products were then ligated into the pGEM-T Easy vector (Promega, Madison, WI) and used to transform DH5 $\alpha$ Max Efficiency competent E. coli (Invitrogen). A minimum of 100 insert-containing colonies were selected from each sample for sequencing and analysis. Sequence analysis of subcloned TCRB gene products was conducted with Sequencher version 4.9 (Gene Codes, Ann Arbor, MI) as previously described. ${ }^{13}$ We have used IMGT nomenclature to assign sequences specific to TCRBV and TCRBJ genes.

SUPPLEMENTARY MATERIAL is linked to the online version of the paper at http://www.nature.com/mi

\section{ACKNOWLEDGMENTS}

This work was supported by the Center for HIV/AIDS Vaccine Immunology, NIAID grant Al067854 and by NIH contract HHSN272201000028C.

\section{DISCLOSURE}

The author declared no conflict of interest.

C 2013 Society for Mucosal Immunology

\section{REFERENCES}

1. Belyakov, I.M., Isakov, D., Zhu, Q., Dzutsev, A. \& Berzofsky, J.A. A novel functional CTL avidity/activity compartmentalization to the site of mucosal immunization contributes to protection of macaques against simian/ human immunodeficiency viral depletion of mucosal CD4+ T cells. J. Immunol. 178, 7211-7221 (2007).

2. Belyakov, I.M. et al. Mucosal AIDS vaccine reduces disease and viral load in gut reservoir and blood after mucosal infection of macaques. Nat. Med. 7, 1320-1326 (2001).

3. Hansen, S.G. et al. Effector memory T cell responses are associated with protection of rhesus monkeys from mucosal simian immunodeficiency virus challenge. Nat. Med. 15, 293-299 (2009).

4. O'Brien, K.L. et al. Adenovirus-specific immunity after immunization with an Ad5 HIV-1 vaccine candidate in humans. Nat. Med. 15, 873-875 (2009).

5. Tatsis, N. et al. Multiple immunizations with adenovirus and MVA vectors improve CD8+ T cell functionality and mucosal homing. Virology 367, 156-167 (2007).

6. Kaufman, D.R. et al. Trafficking of antigen-specific CD8+ T lymphocytes to mucosal surfaces following intramuscular vaccination. J. Immunol. 181, 4188-4198 (2008)

7. Schmitz, J.E. et al. Control of viremia in simian immunodeficiency virus infection by CD8+ lymphocytes. Science 283, 857-860 (1999).

8. Barouch, D.H. et al. Eventual AIDS vaccine failure in a rhesus monkey by viral escape from cytotoxic T lymphocytes. Nature 415, 335-339 (2002).

9. Price, D.A. et al. T cell receptor recognition motifs govern immune escape patterns in acute SIV infection. Immunity 21, 793-803 (2004).

10. Messaoudi, I., Guevara Patino, J.A., Dyall, R., LeMaoult, J. \& NikolichZugich, J. Direct link between mhc polymorphism, T cell avidity, and diversity in immune defense. Science 298, 1797-1800 (2002). 
11. Turner, S.J., La Gruta, N.L., Kedzierska, K., Thomas, P.G. \& Doherty, P.C. Functional implications of T cell receptor diversity. Curr. Opin. Immunol. 21, 286-290 (2009).

12. Meyer-Olson, D. et al. Limited T cell receptor diversity of HCV-specific T cell responses is associated with CTL escape. J. Exp. Med. 200, 307-319 (2004).

13. Sircar, P., Furr, K.L., Dorosh, L.A. \& Letvin, N.L. Clonal repertoires of virusspecific CD8+ T lymphocytes are shared in mucosal and systemic compartments during chronic simian immunodeficiency virus infection in rhesus monkeys. J. Immunol. 185, 2191-2199 (2010).

14. Rudd, B.D. et al. Diversity of the CD8+ T cell repertoire elicited against an immunodominant epitope does not depend on the context of infection. J. Immunol. 184, 2958-2965 (2010).

15. Price, D.A. et al. Public clonotype usage identifies protective Gag-specific CD8+ T cell responses in SIV infection. J. Exp. Med. 206, 923-936 (2009).

16. Smith, M.Z. et al. Limited maintenance of vaccine-induced simian immunodeficiency virus-specific CD8 T-cell receptor clonotypes after virus challenge. J. Virol. 82, 7357-7368 (2008).

17. Gallichan, W.S. \& Rosenthal, K.L. Long-lived cytotoxic T lymphocyte memory in mucosal tissues after mucosal but not systemic immunization. J. Exp. Med. 184, 1879-1890 (1996).

18. Mattapallil, J.J. et al. Vaccination preserves CD4 memory T cells during acute simian immunodeficiency virus challenge. J. Exp. Med. 203, 1533-1541 (2006).

19. Koelle, D.M. et al. Expression of cutaneous lymphocyte-associated antigen by $\mathrm{CD} 8(+) T$ cells specific for a skin-tropic virus. J. Clin. Invest. $110,537-548$ (2002).

20. Mora, J.R. et al. Selective imprinting of gut-homing T cells by Peyer's patch dendritic cells. Nature 424, 88-93 (2003).

21. Mora, J.R. et al. Reciprocal and dynamic control of CD8T cell homing by dendritic cells from skin- and gut-associated lymphoid tissues. J. Exp. Med. 201, 303-316 (2005).

22. Gallichan, W.S. \& Rosenthal, K.L. Long-term immunity and protection against herpes simplex virus type 2 in the murine female genital tract after mucosal but not systemic immunization. J. Infect. Dis. 177, 1155-1161 (1998).

23. Belyakov, I.M. et al. Mucosal immunization with HIV-1 peptide vaccine induces mucosal and systemic cytotoxic T lymphocytes and protective immunity in mice against intrarectal recombinant HIV-vaccinia challenge. Proc. Natl. Acad. Sci. USA 95, 1709-1714 (1998).
24. Stevceva, L. et al. Both mucosal and systemic routes of immunization with the live, attenuated NYVAC/simian immunodeficiency virus SIV(gpe) recombinant vaccine result in gag-specific CD8(+) T-cell responses in mucosal tissues of macaques. J. Virol. 76, 11659-11676 (2002).

25. Masopust, D. et al. Activated primary and memory CD8T cells migrate to nonlymphoid tissues regardless of site of activation or tissue of origin. J. Immunol. 172, 4875-4882 (2004).

26. Masopust, D. et al. Dynamic T cell migration program provides resident memory within intestinal epithelium. J. Exp. Med. 207, 553-564 (2010).

27. Liu, L., Fuhlbrigge, R.C., Karibian, K., Tian, T. \& Kupper, T.S. Dynamic programing of CD8+ $T$ cell trafficking after live viral immunization. Immunity 25, 511-520 (2006).

28. Masopust, D., Vezys, V., Wherry, E.J., Barber, D.L. \& Ahmed, R. Cutting edge: gut microenvironment promotes differentiation of a unique memory CD8T cell population. J. Immunol. 176, 2079-2083 (2006).

29. Klonowski, K.D. et al. Dynamics of blood-borne CD8 memory T cell migration in vivo. Immunity 20, 551-562 (2004).

30. Debes, G.F. et al. Chemokine receptor CCR7 required for T lymphocyte exit from peripheral tissues. Nat. Immunol. 6, 889-894 (2005).

31. Bromley, S.K., Thomas, S.Y. \& Luster, A.D. Chemokine receptor CCR7 guides T cell exit from peripheral tissues and entry into afferent lymphatics. Nat. Immunol. 6, 895-901 (2005).

32. Zammit, D.J., Turner, D.L., Klonowski, K.D., Lefrancois, L. \& Cauley, L.S. Residual antigen presentation after influenza virus infection affects CD8T cell activation and migration. Immunity 24, 439-449 (2006).

33. Ely, K.H., Cookenham, T., Roberts, A.D. \& Woodland, D.L. Memory T cell populations in the lung airways are maintained by continual recruitment. J. Immunol. 176, 537-543 (2006).

34. Hansen, S.G. et al. Profound early control of highly pathogenic SIV by an effector memory T-cell vaccine. Nature 473, 523-527 (2011).

35. Masopust, D., Vezys, V., Marzo, A.L. \& Lefrancois, L. Preferential localization of effector memory cells in nonlymphoid tissue. Science $\mathbf{2 9 1}$, 2413-2417 (2001).

36. Wakim, L.M., Woodward-Davis, A. \& Bevan, M.J. Memory T cells persisting within the brain after local infection show functional adaptations to their tissue of residence. Proc. Natl. Acad. Sci. USA 107, 17872-17879 (2010).

37. Shacklett, B.L., Critchfield, J.W. \& Lemongello, D. Isolating mucosal lymphocytes from biopsy tissue for cellular immunology assays. Methods Mol. Biol. 485, 347-356 (2009). 